\title{
Study on the Mechanism and Application of Applying Magnetic Barkhausen Noise to Evaluate Dislocation Density and Plastic Deformation
}

\author{
Xueliang Kang, ${ }^{\mathrm{a}, \mathrm{b}}$, Shiyun Dong ${ }^{\mathrm{a}}$, Hongbin Wang ${ }^{\mathrm{b}, 1}$, Xiaoting Liu ${ }^{\mathrm{a}}$, Shixing Yan \\ a National Key Laboratory for Remanufacturing, Army Academy of Armored Forces, \\ Beijing 100072, China \\ ${ }^{\mathrm{b}}$ School of Materials Science and Engineering, Shanghai University, Shanghai 200072, \\ China
}

\begin{abstract}
Seven specimens of 45 steel with different residual strains were prepared by homogeneous plastic tensile test. The microstructure of the specimens was observed by scanning electron microscopy and the texture characteristics of the specimens were studied by X-ray diffraction. The results showed that plastic deformation mainly leads to dislocation increment in the microstructure rather than obvious deformed grain morphology, texture and residual stress. Then the dislocation density of each sample was calculated by X-ray diffraction method. The MBN signals of the samples were tested by magnetic Barkhausen noise method and the corresponding RMS (root mean square) values were calculated. The results showed that the dislocation density increases and the RMS value decreases with the increase of plastic deformation magnitude, the phenomenon was explained deeply. By establishing the correlation between dislocation density and RMS value, it was found that there was a good linear relationship between dislocation density and RMS value. According to the formula provided by the fitting curve, the dislocation density can be predicted by measuring the RMS value of any degree of plastic deformation.
\end{abstract}

Keywords: magnetic Barkhausen noise; dislocation density; plastic deformation; quantitative evaluation

\section{Introduction}

Magnetic Barkhausen noise (MBN) is a nondestructive testing technology that is widely used in detection of thickness of carburization layer ${ }^{[1]}$ and film ${ }^{[2]}$, grain size $^{[3]}$, phase content ${ }^{[4,5]}$, stress ${ }^{[6,7]}$.etc. This phenomenon is due to the interactions of domain walls with defects like grain boundaries, dislocations, inclusions and second phases, so the MBN is sensitive to microstructural changes of ferromagnetic materials. During plastic deformation, microstructure of the material will change significantly, so the MBN method can be used for characterizing plastic deformation.

Pedro P. de C. Antonio et al studied the variation of MBN signal under small plastic deformation, he attributed the change of MBN signal to the influence of many factors ${ }^{[8]}$.

${ }^{1}$ Corresponding author: kangxueliangyx@163.com, syd422@sohu.com. 
Martin J. Sablik et al tried to model the stress-strain curve with a modified theory, he thought that the $90^{\circ}$ domain wall would also cause the change of MBN signal, but no further discussion was given ${ }^{[9]}$. The research in this field is rare, and the experiment is basically carried out under the action of coaxial external force, so the influence of external force is introduced ${ }^{[10,11]}$. In order to study the influence of dislocation density on MBN signal separately, a special design and demonstration was carried out in this study.

Dislocation is a common type of lattice defect in crystal materials, which is strongly related to mechanical properties of metals, the four theories of strength for materials are all based on dislocation theory. Dislocation has always been a hot topic in the research of metallic materials. The microscopic mechanism of plastic deformation can be explained by dislocation theory ${ }^{[12,13]}$, Dislocation density determines the process of recovery and recrystallization ${ }^{[14-17]}$. Dislocation density plays a key role in work-hardening of materials ${ }^{[18-20]}$. In a word, dislocation provides an important characterization method for material science research.

Dislocation density is the main parameter for characterizing dislocation. There are several commonly used methods of calculating dislocation density, among them TEM and X-ray diffraction line profile analysis are the most widely used techniques to quantify the dislocation density. Each analytical method has its characteristics, application range and limitations. TEM technique can give an in-situ observation of dislocation morphology ${ }^{[21,22]}$, however it is time consuming for a TEM sample preparation, and obtained information is from small area. Besides, for a sample with large number of dislocations, it is difficult to distinguish one from another, so TEM is mainly used for low density dislocation research. XRD reveals the average data over a relatively large area and it can be used for high density dislocation research, however, the sample preparation and observation is complex as well.

Detection of dislocation density by non-destructive method is free of the complicated sample preparation process, which is facilitate for life prediction ${ }^{[23]}$ and damage assessment $^{[24]}$. In this paper, seven specimens of 45 steel with different residual strains were prepared by homogeneous plastic tensile test. The microstructure of the specimens was observed by scanning electron microscopy and the texture characteristics of the specimens were studied by X-ray diffraction. Then the dislocation density of each sample was calculated by X-ray diffraction method. The MBN signals of the samples were tested by magnetic Barkhausen noise method and the corresponding RMS (root mean square) values were calculated.

\section{Materials and methods}

\subsection{Material preparations}

Tensile specimens of the same size were prepared with 45 steel. The samples were annealed to eliminate residual stress. In order to obtain different dislocation density, the tensile method was adopted. Three specimens were stretched to break, and the stress-strain curves were obtained. The mean maximum strain was about $21 \%$. Then 7 specimens with different residual strains were obtained by tensile method (Fig.1), the residual strains were $1 \%, 4 \%, 8 \%, 12 \%, 14 \%, 16 \%$, and $18 \%$ respectively, the specimens were named as \#1, \#2, \#3, \#4, \#5, \#6, \#7 correspondingly. 


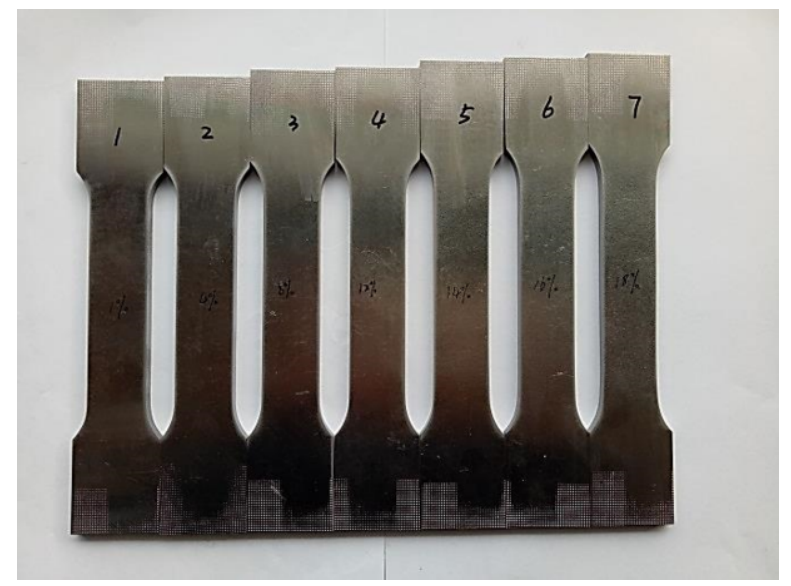

Figure 1. Tensile specimens with different plastic deformation

\subsection{MBN measurements}

MBN measurement was performed using the experimental system developed in the authors' laboratory. The test system is schematically shown in fig. 2.

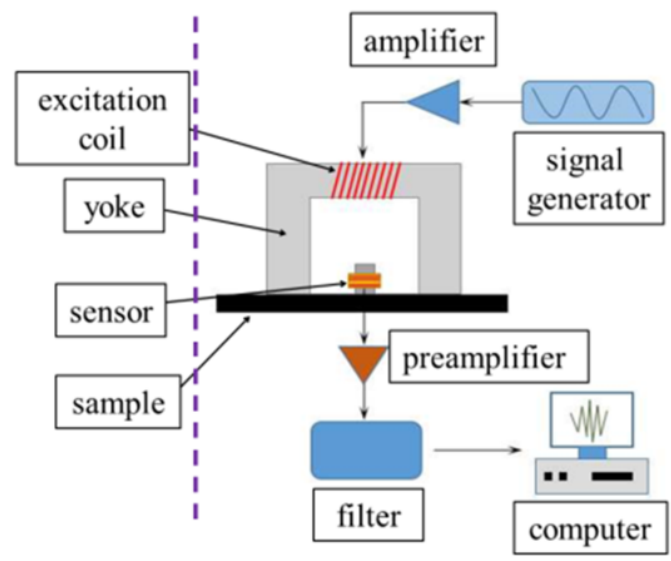

Figure 2. Experimental set-up of $\mathrm{MBN}$

The U-shaped yoke placed on the surface of the sample is used to provide a magnetic field. A sinusoidal signal provided by the function generator is applied to the excitation coil. The pick-up coil used to detect the MBN signals is mounted in the middle between the legs of the U-core. The original signal is amplified and filtered. To minimize undesirable effect of eddy current in the induction process, in the experiment the excitation frequency was set to $10 \mathrm{~Hz}$, the sampling frequency was set to $200 \mathrm{kHz}$.

The morphology of one MBN signal is shown in figure 3. The MBN signal is composed of many pulses of different intensities. In order to quantitatively describe one MBN signal, the RMS (root mean square) is used as the eigenvalue. The RMS voltage of the MBN signal is computed using the expression 


$$
\mathrm{V}_{\mathrm{RMS}}=\sqrt{\left(\sum_{\mathrm{i}} \mathrm{V}_{\mathrm{i}}^{2}\right) / \mathrm{n}}
$$

Where $V_{i}$ is voltage intensity and $n$ is the number of voltage pulses.

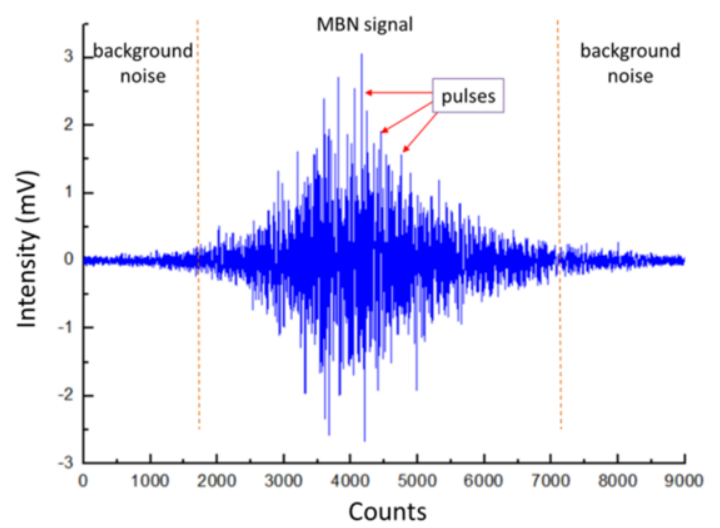

Figure 3. Morphology of one MBN signal

\subsection{Microstructure characterization and X-ray Diffraction Measurements}

The microstructure of the specimen was characterized by scanning electron microscope (SEM). The specimens were prepared according to the standard procedure, etched with Nitric acid alcohol solution (4\% HNO3, 96\% alcohol) after grinding and polishing. A PANalytical X-Pert system with $\mathrm{Cu}-\mathrm{k}_{\alpha}$ radiation $(\lambda=0.154 \mathrm{~nm})$ was used for XRD measurement. The X-ray tube was operated at $40 \mathrm{kV}$ and $40 \mathrm{~mA}$. The diffraction lines were recorded from $2 \theta=10^{\circ}$ to $90^{\circ}$ with a step of $0.02^{\circ}$ to cover the main diffraction angles of the samples. The (110), (200) and (211) pole figures were measured and the orientation distribution function (ODF) was calculated for texture analysis.

\section{Results and discussion}

\subsection{Microstructure analysis}

The samples for MBN and XRD measurement were free from external influences (Force, temperature and magnetic field), the microstructure completely determines the MBN and XRD signals. The influence of microstructure can be divided into different factors, such as dislocation, residual stress, grain size, texture and so on. The external force was uniformly and slowly applied to the cross section of the specimen during tensile test, resulting in uniform plastic deformation in the material, no macroscopic residual stress was generated in the specimens. Texture is another important factor affecting the detection signal. Texture may be formed during plastic deformation, Textural structure with specific orientation distribution results in anisotropy of the detection signals. Plastic deformation can also cause grain shape changes, the size of which depends on the composition of the material, the type of microstructure and the degree of plastic deformation. The morphology and size of the grains will also cause 
changes in the detection signals. The microstructure and texture of the specimens were observed.
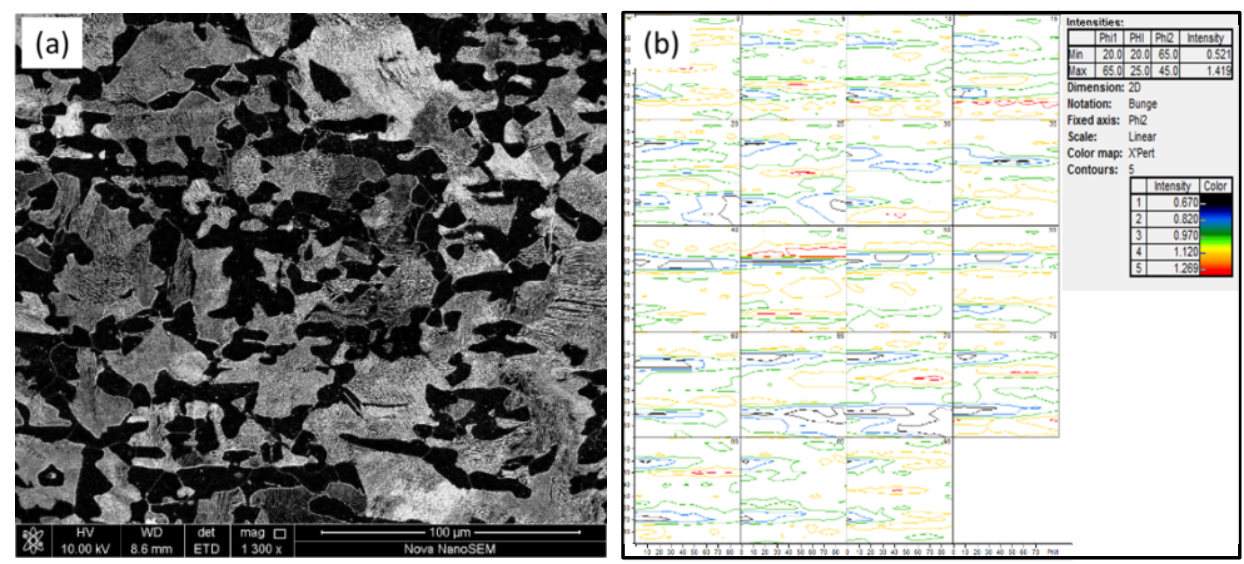

(a)SEM figure; (b) ODF figure

Figure 4. microstructure and texture observation of the \#7 specimen

The corresponding result of the \#7 specimen was given in figure 4. As shown in Fig.4 (a), the ferrite (black in color) and pearlite (white in color) is uniformly distributed, no obvious deformed structures or bands were observed. Fig.4 (b) shows the orientation density map at different directions. According to the color scale, the orientation density is uniformly distributed in all directions, and there is no orientation concentration in the material. The deformation of the \#7 specimen is the largest, and there was no deformed microstructure and texture produced during tensile deformation. The test results of other samples with smaller deformation are similar. In summary, tensile deformation process mainly results in changes of dislocation density of the specimens.

\subsection{Dislocation density measured by X-ray diffraction method}

The dislocation density was measured according to the XRD profile analysis method. The modified Williamson-Hall method $(\mathrm{m}-\mathrm{WH})$ is a widely used method to evaluate the dislocation density[25,26]. The dislocation density measured by X-ray diffraction experiment can be described by the following equation[27]:

$$
\begin{gathered}
(\Delta \mathrm{K})^{2}=\left(\frac{\alpha}{\mathrm{d}}\right)^{2}+\beta \mathrm{K}^{2} \mathrm{C}+\mathrm{O}\left(\mathrm{K}^{2} \mathrm{C}^{2}\right) \\
\Delta \mathrm{K}=\frac{2 \mathrm{~W} \cos \theta}{\lambda} \\
\mathrm{K}=\frac{2 \sin \theta}{\lambda} \\
\beta=\frac{\pi \mathrm{M}^{2} \mathrm{~b}^{2}}{2} \rho
\end{gathered}
$$

In which, $\alpha$ is shape factor, $\mathrm{d}$ is the average grain size, $\theta$ is diffraction angle, $\mathrm{W}$ is the full-width-half-maximum (FWHM) of the XRD diffraction peak, $\rho$ is the dislocation density, $\lambda$ is $\mathrm{X}$-ray wave length, $\mathrm{K}$ is the magnitude of diffraction vector, 
$\mathrm{M}$ is a constant parameter depending on the effective outer cutoff radius of dislocation, in deformed materials $M$ varies in between 1 and 2, for most instance $M$ was selected as $2[28,29]$, $\mathrm{O}$ indicates non-interpreted higher order terms, $\mathrm{b}$ is the magnitude of the Burgers vector. $\mathrm{C}$ is the so called dislocation contrast factor, which is determined by the elastic anisotropy and the dislocation type of the material

$$
\mathrm{C}=\mathrm{C}_{\mathrm{h} 00}^{\prime}\left(1-\mathrm{qH}^{2}\right)
$$

$\mathrm{C}_{\text {h00 }}^{\prime}$ is a constant $(0.258)$. In a similar manner, the q parameter was determined as 1.977. $\mathrm{H}^{2}$ can be expressed as the fourth-order invariant function of the Miller indices (hkl):

$$
\mathrm{H}^{2}=\frac{\mathrm{h}^{2} \mathrm{k}^{2}+\mathrm{k}^{2} \mathrm{l}^{2}+\mathrm{l}^{2} \mathrm{~h}^{2}}{\left(\mathrm{~h}^{2}+\mathrm{k}^{2}+\mathrm{l}^{2}\right)^{2}}
$$

According to Eq. (2), the $\Delta \mathrm{K}$ for each (hkl) plane was plotted as a function of $\mathrm{K}^{2} \mathrm{C}^{2}$, after linear fitting, the slope $\beta$ was calculated and the dislocation density $\rho$ can be obtained according to Eq. (5).

In this research, $b=0.284[31], \alpha$ is given as 0.9 under assumption of spherical crystals with cubic symmetry[32], the results of X-ray diffraction was given in figure 5 .

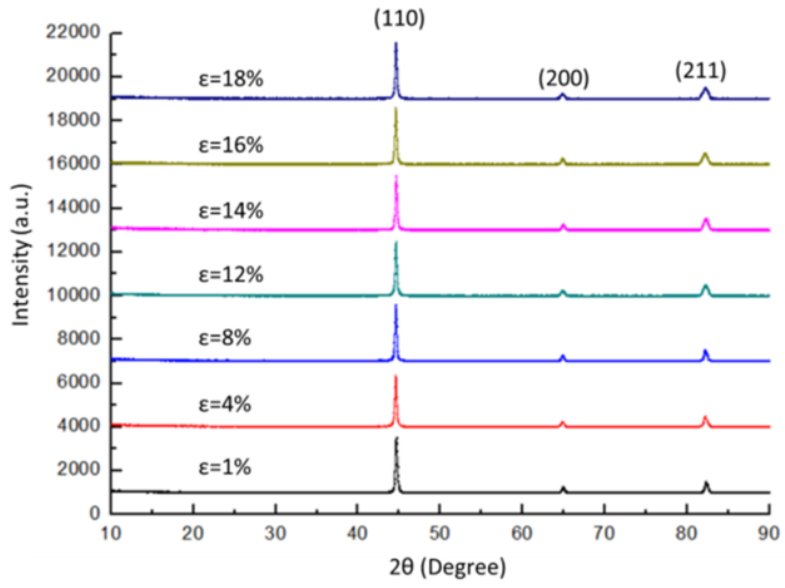

Figure 5. XRD diffraction peaks of the specimens

As shown in figure 5, 3 distinct diffraction peaks ((110), (200) and (211)) were detected in each sample. The diffraction peaks have obvious broadening characteristics. FWHM values of the (110), (200) and (211) X-ray diffraction peaks were obtained from the original diffraction data. However for (h00) plane, the value of $\mathrm{H}^{2}$ in Eq. (7) is zero. In such a case Eq. (2) cannot be applied[33], so only diffraction data from (110) and (211) was used. The relationship between $(\Delta \mathrm{K})^{2}$ and $\mathrm{K}^{2} \mathrm{C}$ was given in Figure 6. After linear fitting, it can be seen clearly in Figure 6 that with the increase of plastic deformation, the slope of fitting curve is increasing. The $\beta$ value of each sample was obtained through fitting and the corresponding dislocation density was calculated according to Eq. (4), as shown in table 1, the dislocation density increases with the increase of plastic deformation. 


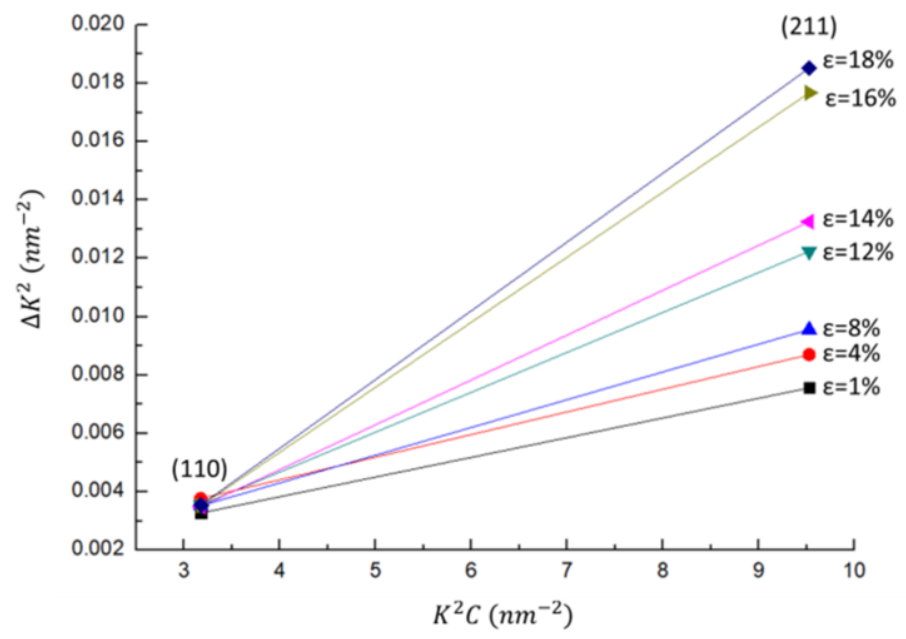

Figure 6. Peak broadening analysis using the modified Williamson-Hall plot

Table 1. Summary of the Coefficient of the Fitting Curve in the Modified Williamson-Hall Plot

\begin{tabular}{cccccccc}
\hline specimen & $\# 1$ & $\# 2$ & $\# 3$ & $\# 4$ & $\# 5$ & $\# 6$ & $\# 7$ \\
\hline Residual strain $(\%)$ & 1 & 4 & 8 & 12 & 14 & 16 & 18 \\
\hline$\beta$ & 0.113 & 0.143 & 0.172 & 0.228 & 0.25 & 0.328 & 0.341 \\
\hline$\rho\left(\times 10^{15} / \mathrm{m}^{2}\right)$ & 2.2 & 2.8 & 3.4 & 4.5 & 4.9 & 6.5 & 6.7 \\
\hline
\end{tabular}

\subsection{Dislocation density evaluated by $M B N$}

After the XRD test, the MBN signals of each sample were tested at the same location. All tests were carried out under the same condition. Figure 7 shows the morphology of 3 MBN signals obtained from each sample. As can be seen clearly, the morphology of each MBN signal is spindle shaped, large in the middle, and small in the two ends. The distribution area of MBN signal corresponding to the plastic deformation of $1 \%$ is the largest, indicating that the MBN signal intensity is the strongest of the 7 specimens, whereas the distribution area of MBN signal corresponding to the plastic deformation of $18 \%$ is the smallest, indicating the weakest intensity of MBN signals. It looks as if the difference of MBN signal morphology is not obvious between the two specimens with similar degree of plastic deformation, on the whole, the MBN signal morphology of the 7 samples is quite different. The MBN signal intensity of specimens from the smallest plastic deformation to the largest plastic deformation is gradually decreasing. In order to assess this difference quantitatively, RMS of each sample was calculated. Because the overall appearance of MBN signals obtained from the same sample is different. The RMS value of each sample is averaged over ten MBN signals to minimize the effect of signal morphological differences.

From macroscopic view, the difference of MBN signals in different samples is caused by plastic deformation. In fact it is the difference of dislocation density that results in the different intensity of MBN signals. Fig. 8 shows the relationship between 
plastic deformation, dislocation density and MBN signal among different specimens. As can be seen clearly, with the increase of plastic deformation degree, the basic rule is the increase of dislocation density and the decrease of MBN signal intensity.

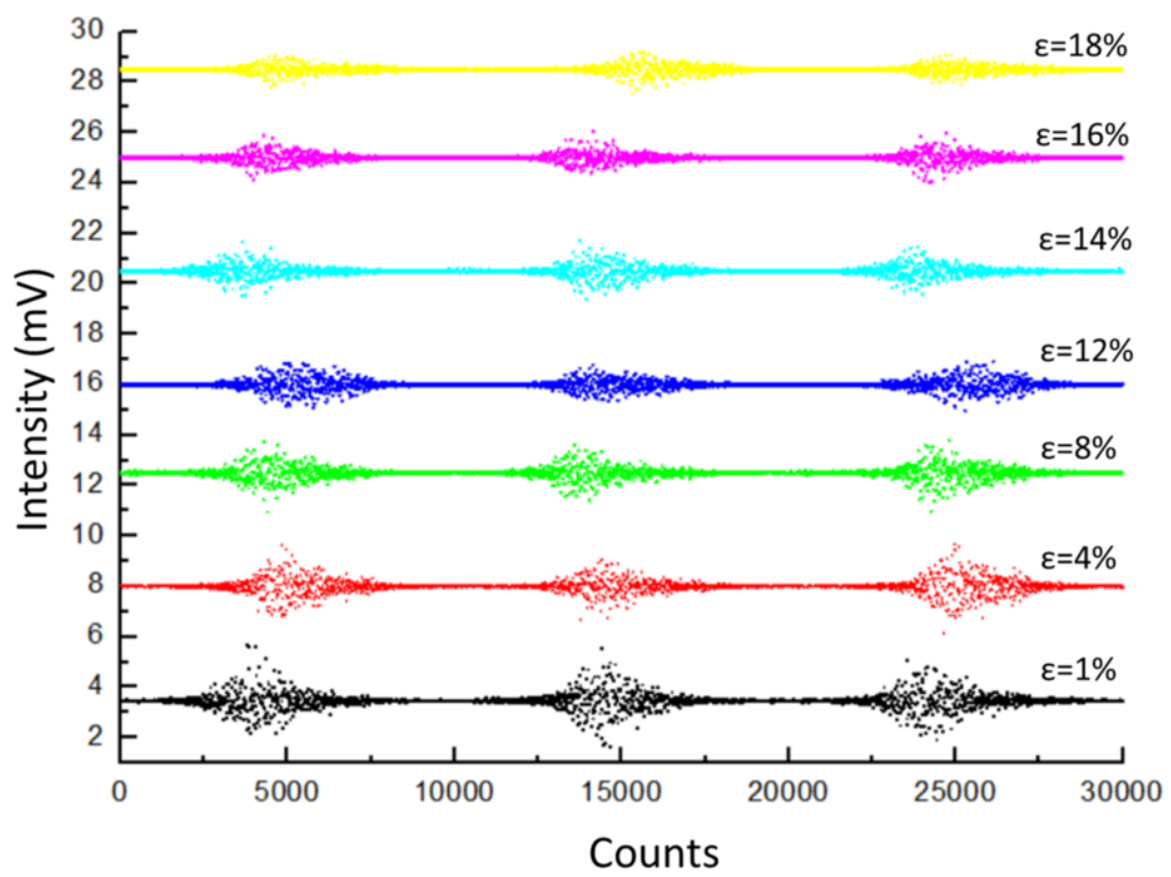

Figure 7. MBN signals of the samples

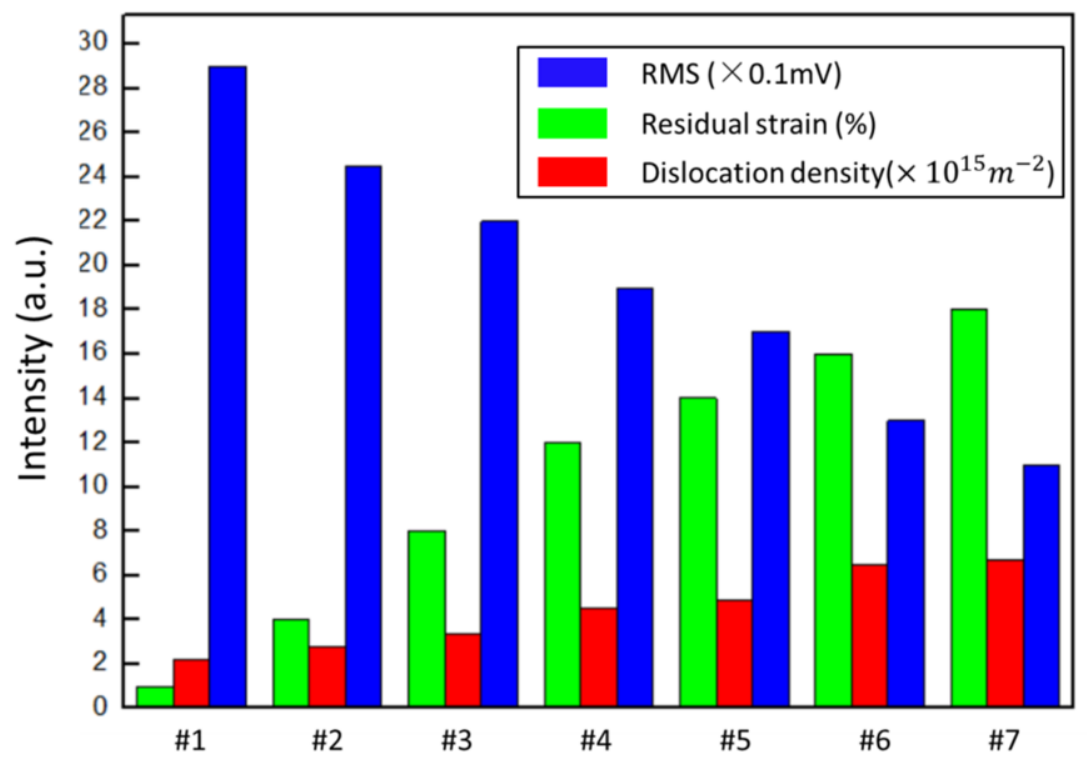

Figure 8. Variation of RMS and dislocation density with the increase of plastic deformation degree 


\subsection{Relationship between dislocation density and MBN signal}

The value of the dislocation density $\rho$ can be calculated from the average values of the crystallite size D and microstrain $\varepsilon$ by relationship[34]

$$
\rho=\frac{3 \sqrt{2 \pi}\left\langle\varepsilon^{2}\right\rangle^{1 / 2}}{\mathrm{Db}}
$$

where $b$ is the Burgers vector.

According to Eq. (8), the dislocation density increases with the increase of plastic strain, the research in this paper has also reached the same conclusion. The dislocation multiplication can be well explained by the Frank-Read source model, which is usually used to characterize dislocation generation in the bulk of a crystalline material[35].

The MBN signal intensity decreases with the increase of dislocation density, this is determined by magnetic domain dynamics. The MBN signal is generated when the magnetic domain moves. Any factor that hinders the movement of magnetic domains will affect the MBN signal. Dislocations hinder the movement of magnetic domain walls. Each dislocation will have a critical value of the pinning force per unite length, the domain wall can move only when the external force is greater than the pinning force provided by all the dislocations. The critical field is defined as[36]

$$
\mathrm{H}_{\text {crit }}=\frac{\gamma \cos \emptyset_{\text {crit }}}{\mu_{0} \mathrm{M}_{\mathrm{S}} \mathrm{L}\left(\cos \Omega_{1}-\cos \Omega_{2}\right)}
$$

Where $\mathrm{L}$ is the distance between two adjacent pinning sites. Under the same test conditions, the other parameters are the same. According to Eq. (9), the critical strength $\mathrm{H}_{\text {crit }}$ for MBN activity decreases as the spacing L increases. According to the model of Nes and Marthinsen[37,38], the slip length of dislocations reduced due to the formation of cell structures and sub-boundaries during deformation. As the degree of deformation increases, the dislocation density increases, the value of $\mathrm{L}$ decreases, the critical field strength required for magnetic domain wall motion increases, and the number of magnetic domains that can be moved decreases, eventually the value of MBN signal decreases.

\subsection{Correlation between dislocation density and MBN signal}

According to the above analysis, the law that the MBN signal decreases with increasing dislocation density is very clear. This makes it possible to quantitatively evaluate the dislocation density using the magnetic Barkhausen noise method, and the relationship diagram was drawn, in which, dislocation density is abscissa and RMS is ordinate, as shown in figure 9. The seven data points show a good linear relationship. According to the regression analysis, the relationship between RMS and Dislocation density can be expressed as:

$$
\mathrm{D}=\frac{\mathrm{R}-3.58}{-0.36}
$$

Where $\mathrm{R}$ indicates RMS intensity, D indicates dislocation density. In order to verify the reliability of the method, three tensile specimens were prepared using the same material and method. The residual strain of the samples was $15 \%$ after tensile deformation. The mean RMS value of the three samples is 1.57, substitute it into Eq. (10), the calculated dislocation density value is $5.58 \times 10^{15} / \mathrm{m}^{2}$. The mean dislocation density of the three specimens tested by the m-WH method was $5.32 \times 10^{15} / \mathrm{m}^{2}$, the 
relative error of this method is $4.9 \%$. The results show that the MBN method has high accuracy in measuring dislocation density.

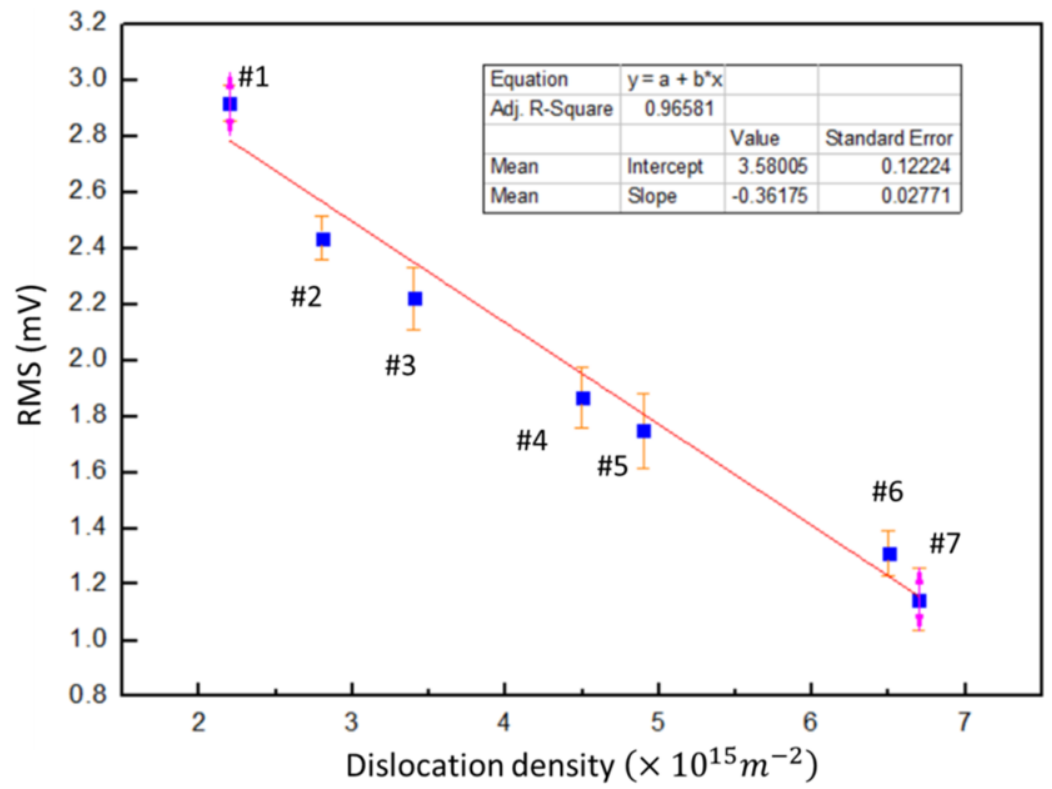

Figure 9. Relationship between RMS and dislocation density

3.6 Correlation between plastic deformation and MBN signal

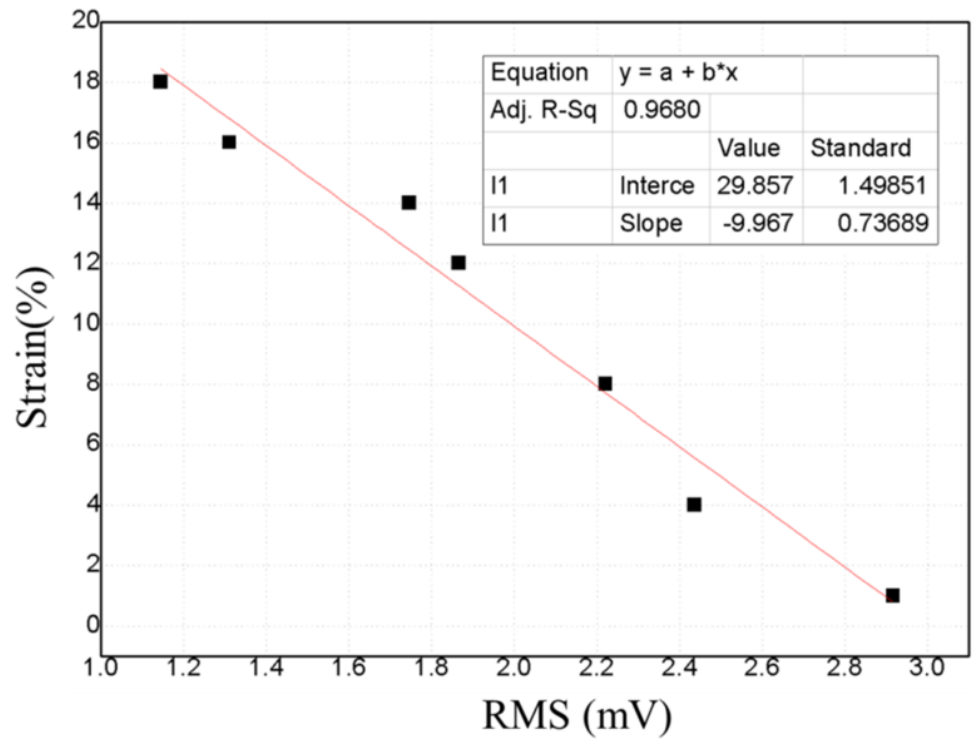

Figure 10. Relationship between RMS and plastic deformation 
There is a negative correlation between plastic deformation and MBN signal, as shown in figure 10, in which, RMS is abscissa and plastic deformation is ordinate. Therefore, MBN signal can be used to predict plastic deformation, and the bridge between them is dislocation density, as discussed above.

\section{Conclusions}

The mainly change of the microstructure of the specimens during tensile deformation is the dislocation multiplication. With the increase of plastic deformation degree, the dislocation density increases and the MBN signal intensity decreases. There are remarkable negative linear relevant relations between RMS and dislocation density as well as plastic deformation. According to the regression function, the dislocation density and plastic deformation can be forecasted, and the prediction value is of high precision. This study confirms that it is feasible to quantitatively evaluate dislocation density and plastic deformation using MBN method.

\section{References}

[1] Zhang M L. Study of Magnetic Barkhausen Noise on the evaluation of case depth on carburized steel [J]. Journal of the American College of Cardiology, 2015, 62(11):981-989.

[2] Seemuang N, Slatter T. Using Barkhausen noise to measure coating depth of coated high - speed steel [J]. International Journal of Advanced Manufacturing Technology, 2017(20):1-12.

[3] Ghanei S, Kashefi M, Mazinani M. Comparative study of eddy current and Barkhausen noise nondestructive testing methods in microstructural examination of ferrite-martensite dual-phase steel [J]. Journal of Magnetism \& Magnetic Materials, 2014, 356(356):103-110.

[4] Ghanei S, Alam A S, Kashefi M, et al. Nondestructive characterization of microstructure and mechanical properties of intercritically annealed dual-phase steel by magnetic Barkhausen noise technique [J]. Materials Science \& Engineering A, 2014, 607(607):253-260.

[5] Vashista M, Moorthy V. On the shape of the magnetic Barkhausen noise profile for better revelation of the effect of microstructures on the magnetisation process in ferritic steels [J]. Journal of Magnetism \& Magnetic Materials, 2015, 393:584-592.

[6] Blaow M, Evans J T, B.A. Shaw. Magnetic Barkhausen noise: the influence of microstructure and deformation in bending [J]. Acta Materialia, 2005, 53(2):279-287.

[7] Sheikh Amiri M, Thielen M, Rabung M, et al. On the role of crystal and stress anisotropy in magnetic Barkhausen noise [J]. Journal of Magnetism \& Magnetic Materials, 2014, 372(12):16-22.

[8] De C A P P, De Campos M F, S Dias F M D, et al. Sharp Increase of Hysteresis Area Due to Small Plastic Deformation Studied With Magnetic Barkhausen Noise[J]. Magnetics IEEE Transactions on, 2014, 50(4):1-4.

[9] Sablik M J, Augustyniak B, De Campos M F, et al. Modeling of Effect of Plastic Deformation on Barkhausen Noise and Magnetoacoustic Emission in Iron With 2\% Silicon[J]. IEEE Transactions on Magnetics, 2008, 44(11):3221-3224.

[10] Dhar A, Clapham L, Atherton D L. Influence of uniaxial plastic deformation on magnetic Barkhausen noise in steel[J]. Ndt \& E International, 2001, 34(8):507-514.

[11] Stefanita C G, Atherton D L., Clapham L, et al. Plastic versus elastic deformation effects on magnetic barkhausen noise in steel[J]. Acta Materialia, 2000, 48(13):3545-3551.

[12] Chattopadhyay A K, Aifantis E C. Stochastically forced dislocation density distribution in plastic deformation [J]. Phys.rev.e, 2016, 94(2).

[13] Harjo S, Kawasaki T, Tomota Y, et al. Unusual Plastic Deformation Behavior in Lath Martensitic Steel Containing High Dislocation Density [C]// Materials Science Forum. 2017.

[14] Humphreys F J, Hatherly M. Recrystallization and Related Annealing Phenomena (Second Edition) [M]. Elsevier, 2004.

[15] Momeni A, Dehghani K, Ebrahimi G R. Modeling the initiation of dynamic recrystallization using a dynamic recovery model [J]. Journal of Alloys \& Compounds, 2011, 509(39):9387-9393. 
[16] Roucoules C, Pietrzyk M, Hodgson P D. Analysis of work hardening and recrystallization during the hot working of steel using a statistically based internal variable model [J]. Materials Science \& Engineering A, 2003, 339(1):1-9.

[17] Tian B, Lind C, Schafler E, et al. Evolution of microstructures during dynamic recrystallization and dynamic recovery in hot deformed Nimonic 80a [J]. Materials Science \& Engineering A, 2004, 367(1): 198-204.

[18] Prasad G V S S, Goerdeler M, Gottstein G. Work hardening model based on multiple dislocation densities [J]. Materials Science \& Engineering A, 2005, 400(1):231-233.

[19] Lavrentev F F, Pokhil Y A. Relation of dislocation density in different slip systems to work hardening parameters for magnesium crystals [J]. Materials Science \& Engineering, 1975, 18(2):261-270.

[20] Mittra J, Dey G K, Dubey J S, et al. Role of dislocation density in raising the stage II work-hardening rate of Alloy 625 [J]. Materials Science \& Engineering A, 2009, 512(1):87-91.

[21] Zielinski W, Lii M J, Gerberich W W. Crack-tip dislocation emission arrangements for equilibrium - I. In situ, TEM observations of Fe, 2wt\%Si [J]. Acta Metallurgica Et Materialia, 1992, 40(11):2861-2871.

[22] Li N, Wang J, Huang J Y, et al. In situ TEM observations of room temperature dislocation climb at interfaces in nanolayered Al/Nb composites [J]. Scripta Materialia, 2010, 63(4):363-366.

[23] Huang J H, Qian Y Y, Jiang Y H, et al. A dislocation model of shear fatigue damage and life prediction of SMT solder joints under thermal cycles [J]. IEEE Transactions on Components Hybrids \& Manufacturing Technology, 2002, 15(4):553-558.

[24] Pangborn R N, Weissmann S, Kramer I R. Dislocation distribution and prediction of fatigue damage [J]. Metallurgical Transactions A, 1981, 12(1):109-120.

[25] He B B, Hu B, Yen $\mathrm{H} \mathrm{W}$, et al. High dislocation density-induced large ductility in deformed and partitioned steels [J] Science, 2017, 357(6355):1029.

[26] Ungár T, Gubicza J, Hanák P, et al. Densities and character of dislocations and size-distribution of subgrains in deformed metals by X-ray diffraction profile analysis [J]. Materials Science \& Engineering A, 2001, 319(4):274-278.

[27] Woo W, Ungár T, Feng Z, et al. X-Ray and Neutron Diffraction Measurements of Dislocation Density and Subgrain Size in a Friction-Stir-Welded Aluminum Alloy [J]. Metallurgical \& Materials Transactions A, 2010, 41(5):1210-1216.

[28] Renzetti R A, Sandim H R Z, Bolmaro R E, et al. X-ray evaluation of dislocation density in ODS-Eurofer steel [J]. Materials Science \& Engineering A, 2012, 534(2): 142-146.

[29] Seth P P, Das A, Bar H N, et al. Evolution of Dislocation Density During Tensile Deformation of BH220 Steel at Different Pre-strain Conditions [J]. Journal of Materials Engineering \& Performance, 2015, 24(7):2779-2783.

[30] T. Ungár, I. Dragomir, Á. Révész, et al. The contrast factors of dislocations in cubic crystals: the dislocation model of strain anisotropy in practice [J]. Journal of Applied Crystallography, 2010, 32(5):992-1002.

[31] Hajyakbary F, Sietsma J, Böttger A J, et al. An improved X-ray diffraction analysis method to characterize dislocation density in lath martensitic structures [J]. Materials Science \& Engineering A, 2015, 639:208-218.

[32] Langford J I, Wilson A J C. Scherrer after sixty years: A survey and some new results in the determination of crystallite size [J]. Journal of Applied Crystallography, 2010, 11(2):102-113.

[33] Ribárik G, Ungár T. Characterization of the microstructure in random and textured polycrystals and single crystals by diffraction line profile analysis [J]. Materials Science \& Engineering A, 2010, 528(1):112-121.

[34] Dini G, Ueji R, Najafizadeh A, et al. Flow stress analysis of TWIP steel via the XRD measurement of dislocation density [J]. Materials Science \& Engineering A, 2010, 527(10):2759-2763.

[35] Xu S, Xiong L, Chen Y, et al. An analysis of key characteristics of the Frank-Read source process in FCC metals [J]. Journal of the Mechanics \& Physics of Solids, 2016, 96:460-476.

[36] Jiles D C. Dynamics of domain magnetization and the Barkhausen effect [J]. Czechoslovak Journal of Physics, 2000, 50(8):893-924.

[37] Marthinsen K, Nes E. A general model for metal plasticity [J]. Materials Science \& Engineering A, 1997 , 234-236(97):1095-1098.

[38] Erik Nes. Modelling of work hardening and stress saturation in FCC metals [J]. Progress in Materials Science, 1997, 41(3): 129-193. 